\title{
Computing Possible Worlds in THE History OF MODERN ASTRONOMY
}

\author{
OSVALDO PESSOA JR. \\ RAFAELA GESING \\ MARIANA JÓ DE SOUZA \\ Daniel Carlos de Melo Marcílio
}

\begin{abstract}
As part of an ongoing study of causal models in the history of science, a counterfactual scenario in the history of modern astronomy is explored with the aid of computer simulations. After the definition of "linking advance", a possible world involving technological antecedence is described, branching out in 1510, in which the telescope is invented 70 years before its actual construction, at the time in which Fracastoro actually built the first prototelescope. By using the principle of the closest possible world (PCP), we estimate that in this scenario the discovery of the elliptical orbit of Mars would by anticipated by only 28 years. The second part of the paper involves an estimate of the probability of the previous scenario, guided by the principle that the actual world is the mean (PAM) and using computer simulations to create possible worlds in which the time spans between advances is varied according to a gamma distribution function. Taking into account the importance of the use of the diaphragm for the invention of the telescope, the probability that the telescope were built by 1538 for a branching time at 1510 is found to be smaller than 1\%. The work shows that one of the important features of computational simulations in philosophy of science is to serve as a consistency check for the intuitions and speculations of the philosopher.
\end{abstract}

Keywords: Causal model; possible worlds; linking advance; history of the telescope; computational philosophy of science.

If anyone looks through two spectacle lenses, one placed on top of the other, he will see everything much larger and closer.

(Fracastoro 1538, apud Van Helden 1977, p.28).

\section{Introduction}

The metaphysics of possible worlds need not speculate on the whole class of logically possible worlds, but may concentrate on counterfactual worlds that are closer to the actual one. "Causally possible worlds" are obtained by branching off from the actual world at some moment of time, in such a way that the branch differs from the main trunk by a random fluctuation of a certain degree (cf. Pessoa 2009; 2014). This view is akin to the elaborated models studied by McCall (1984).

Principia 20(1): 117-12 (2016).

Published by NEL — Epistemology and Logic Research Group, Federal University of Santa Catarina (UFSC), Brazil. 
A subclass of causally possible worlds which lies close to the actual one refers to possible histories of science in which the time span between causes and effects is varied randomly, resulting in small changes in the "causal model" that describes the history of the scientific field. This subclass is explored in this paper.

Let us define an advance as any contribution made by a scientist, be it in the "right" or "wrong" direction, which is part of scientific activity. Such units of scientific activity may include ideas, explanations, problems, experiments, data, etc. Advances are conceived as being related by causal connections, in the sense that the absence of an advance (the cause) will affect the probability of another advance (the effect) taking place. The historical development of a scientific field may be described by causal models involving advances (cf. Pessoa 2010).

In the present research, a computer program is used to generate counterfactual histories in which the time spans between advances are varied according to a gamma probability distribution (Pessoa 2006). These scenarios conserve the actual causal relation between the advances, since they are generated by a single causal model. However, the change of temporal order in which the advances (in different causal chains) appear may suggest new causal connections, which when introduced by the researcher into the data base constitutes a broader causal model.

\section{Postulating linking advances}

As an example, consider the field of astronomy in the 16th and 17th centuries. Using Tycho Brahe's data on Mars, Johannes Kepler was able to infer in 1605 that its orbit is an ellipse, independently of the invention of the telescope by Hans Lipperhey, in 1608. However, the invention of the telescope could well have happened before. An analysis of the probability of this happening will be given in $\S 4$.

Let us now assume that that there is a causally possible world $W$ that branches off the actual world around 1510, right after the circulation of Nicolas Copernicus' heliocentric ideas in the Commentariolus, and that around 1538 (in $W$ ) the telescope is developed. This is the year, in our actual world, in which Girolamo Fracastoro experimented with spectacles and noticed the magnifying power of a pair of lenses held together (it is not known whether one of them was concave; cf. Van Helden 1977, p.15, p.28). In the counterfactual scenario presented, the early invention of the telescope, 70 years before the actual time, would lead more or less rapidly to the downfall of the Ptolemaic system, given the observations of the phases of Venus. This would strengthen both the Copernican and the Tychonian type systems. More time would be required to show that the orbit of each planet is an ellipse around the Sun placed at a focus (see $\S 3$ below).

From our perspective in $W$, the counterfactual causal connection between the invention of the telescope and the discovery of the elliptical orbit of Mars in world $W$

Principia 20(1): 117-126 (2016). 
is easy to postulate and analyze, since we are imagining a technological antecedence, i.e. a situation in which a powerful technological innovation appears before the time it actually occurred in $W$. We may attribute a high probability in $W$ for the discovery of elliptical orbits before 1600, without the need of imagining any advance that did not happen in our world. One has to consider how the use of the telescope could furnish precise measurements of the positions of Mars, but the advances involved in this path would be very similar to the ones that in fact, in our world $W$, led from the invention of the telescope to a confirmation and extension of Brahe's data.

However, from the perspective of our ancestral counterparts in $W$, the postulation of world $W$ would involve the postponement of a technological advance (the invention of the telescope). Could they have imagined that the elliptical path could have been discovered without the telescope? That would have required a very good knowledge of the history of astronomy. One would have to realize that the construction of large and precise astronomical instruments (such as the ones developed by Brahe) was technologically and socially viable and would give enough accuracy in the measurements of the apparent positions of the planets to allow for the interpolation of an ellipse. Such an advance may be called a linking advance, since, from the perspective of $W$, it is a non-existent advance (assuming that the early development of the telescope in $W$ aborted the construction of large naked eye observatories) that must be postulated for making the connection between the state of art in 1510 (shared by both worlds) and the postulation of elliptical orbits, without the involvement of telescopes.

Reasoning under the supposition of symmetry between $W$ and $W$, we have therefore seen that the postulation of linking advances is necessary in part of the counterfactual scenarios to be imagined in the history of science.

\section{Exploring the antecedence of the invention of the telescope}

As a matter of fact, "Tycho's observations represented the best record of the state of the heavens" (King 1955, p.93) until the $1630 \mathrm{~s}$. Only then did amateur astronomers start improving over his methods of observation: Pierre Vernier, in Burgundy, improved the techniques of reading arcs and circles; Jeremiah Horrocks, from close to Liverpool, used the telescope to give better predictions of the transit of Venus and to show that the lunar orbit is elliptical; Jean-Baptiste Morin (1634) fitted a Galilean telescope to a divided circle and was able to see stars in the daytime; and William Gascoigne, near Leeds, discovered around 1640 how to fit crossed threads between lenses and to use a micrometer in a Keplerian telescope (King 1955, pp.93-7).

Around 32 years elapsed between the invention of the telescope and its coupling to measuring scales in order to improve on Tycho's unaided visual observations in- 


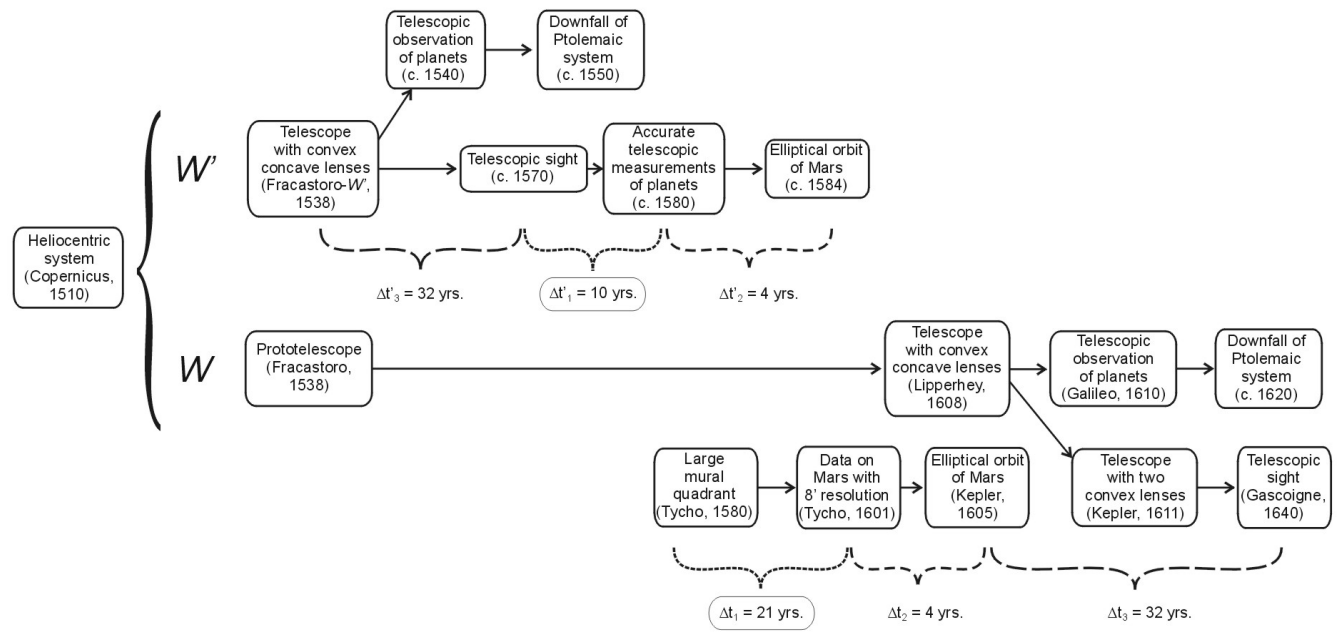

Figure 1: Causal models comparing the history of astronomy in our actual world $W$ and in a counterfactual world $W$ in which the telescope is taken to be invented in 1538. Time spans in the actual world were maintained in $W$, except for $t_{1}$. Although the telescopic observation of planets would be anticipated by 70 years in $W$, leading to the downfall of the Ptolemaic system, the discovery of the elliptical orbit of Mars would be anticipated by only 21 years.

volving accurate measurements of angles (see $t_{3}$ in Fig. 1). It is thus reasonable to assume, in $W$, that the same 32 years would pass between the counterfactual invention of the telescope, stipulated around $1538_{W}$, and the beginning of its use for accurate measurements of Mars' orbit, around $1570_{W}$.

This reasoning involves the principle of projecting onto the counterfactual world the same state of affairs of the factual world, modulo the changes introduced for defining the counterfactual world. In Lewis' metric of possible worlds, this is equivalent to choosing the closest possible world that satisfies the defining conditions. Another way of expressing this is saying that the date of invention of the telescope is changed under the clause of ceteris paribus (i.e., nothing else changes). It is worth noting that the closest possible world (in which the time of a cause is changed) is taken to be the one on which the time span between cause and effect is maintained, and not the one in which the date of the effect remains unchanged.

The "principle of the closest possible world" (PCP) may be violated if there are good reasons for doing so. In Fig. 1, this is done with $t_{1}$, which is the time taken between the construction of the large mural quadrant by Tycho and the end of his collection of data on Mars, a time span of around 21 years. But each calculation of the orbit of Mars done by Kepler uses data of four Martian orbits, each of which has a period of two terrestrial years, so it is reasonable to project $t_{1}=10$ years in $W$, so 
that by $1580_{W}$ the data on Mars would be available. After that, using PCP, $t_{3}=4$ more estimated years would be needed for the discovery that Mars' orbit is elliptical, around $1584_{W}$. This gives an overall antecedence of 21 years in $W$ for the discovery of elliptical orbits.

It should be stressed that when we imagine that an advance occurred 70 years before it actually did, and apply PCP, we are ignoring the different cultural contexts between the two cases, which could have an influence on the time interval for the appearance of the effect.

\section{Invention of the telescope in possible worlds}

In our previous discussion, we took the year 1510 as the branching time $t_{0}$ of a counterfactual history which led to the invention of the telescope in 1538. What would be an estimate for the probability of such an advance happening before 1538, given the state of affairs of the actual world in 1510 ?

In order to estimate this probability, we used a computer program developed by us, called SIMPOSS 1, which generates possible histories of science by modifying the time span between advances connected by a causal relation. Fig. 2 is an example of the probability distribution for time spans generated between two advances connected by a causal relation. The full program generates possible worlds by randomly choosing each time span for every pair of causes and effect in the causal model.

The simulation implements the "principle that the actual world is the mean" (PAM), i.e., the mean value for the effect (the invention of the prototelescope) in all possible worlds is the year in which it took place in the actual world. In Fig. 2a, the actual value is the mean of three independent inventions of the prototelescope, which we took to be the instruments built by Fracastoro (1538), Leonard Digges (1571) and Della Porta (1589) (we left out Bourne's 1578 device); in Fig. 2b we took the actual year of invention to be 1571 (just for comparison with Fig. 2a).

The causal model to be written out should be based on the account of historians of science. Willach (2010, pp.111-3) analyzes the question of why Lipperhey was capable of constructing a practical instrument, with 3 magnification, even though the lenses he used probably had comparable quality to those previously explored in prototelescopes. "If the knowledge of the magnification potential of two combined spectacle glasses was already available many decades before 1608, why was the telescope not invented earlier?" (Willach 2007, apud Zuidervaart 2010, p.40). He concludes that the crucial novelty was the introduction of a diaphragm, that cuts off the light that would suffer aberration when passing through the external part of the lens and allows only the light that passes through the center of the lens to be seen. Only after the techniques for polishing lenses were improved in 1645 by Anton Schryl de Rheita 


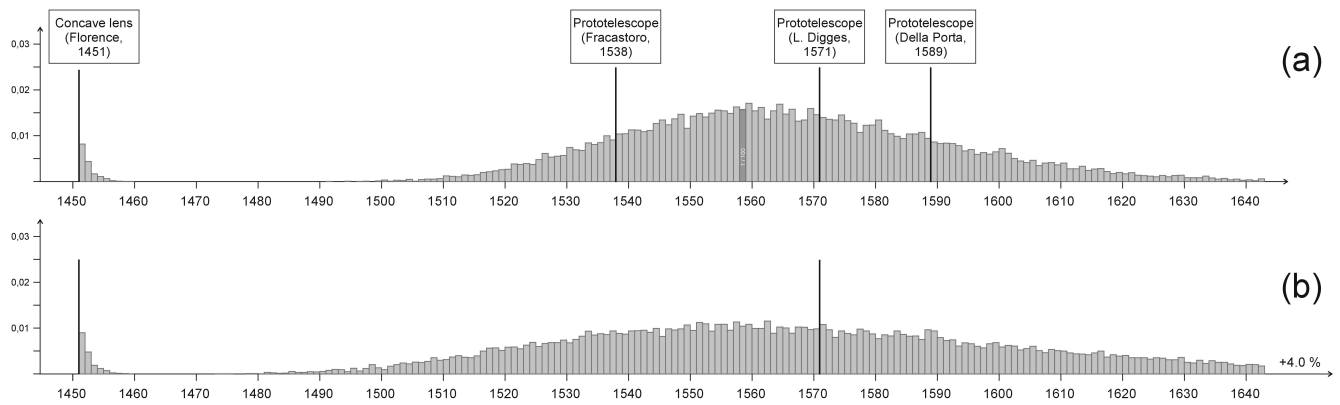

Figure 2: Distribution of time spans between two advances, in which the first (concave lens) is taken to be the cause of the second (prototelescope). The dark rectangle represents one world, in the case 100 worlds are considered. The actual histogram was generated by program SIMPOSS 1, in Python language, for 20,000 possible worlds. (a) Considering three independent appearances of the effect (in 1538, 1571 and 1589), one can estimate the gamma function distribution curve by fitting two parameters (mean and standard deviation) to three data points. The initial kink at 1451 incorporates data beyond 1642. (b) In the case in which there is only one data for the effect, the standard deviation is arbitrarily fixed at $c_{v}=\frac{1}{3}$ of the mean ( $\mathrm{cv}$ is called the coefficient of variation). The resulting distribution is more spread out than the first one, which indicates that cv could be lowered. To the right, $4.0 \%$ of the data are not shown.

and Johannes Wiesel could the telescopes be built without a narrow diaphragm. Van Helden (1977, p.26) also mentions the diaphragm to explain the success of Galileo. Dupré (2003) notes that a diaphragm coupled to a lens had already been used by painters, who used the camera obscura to draw in perspective, and the diaphragm was used to "see an even more vivid effect" (Daniele Barbaro 1568, apud Dupré 2003, p.370).

We thus consider the simplified causal model represented in Fig. 3, representing the invention of the telescope in our actual world. Our aim is to imagine possible worlds branching out in the year 1510, so that before that time they will be identical. Thus, in the model of Fig. 3, the dates of the three advances before 1510 are fixed. We simulated 20,000 possible worlds (one of them being the actual world) in three different ways.

Our initial approach (Fig. 4b) was to run simulations based on Fig. 3 and discard all possible worlds in which either the diaphragm or the prototelescope appears before 1510. The problem here is that the principle that the actual world is the mean (PAM) is not satisfied, as the mean of the simulations is around 1662, not 1608 as in the actual world.

One alternative is simply to consider all initial causes (dioptra, convex and concave lenses) as occurring at the branching time 1510 and run the simulation (as in 


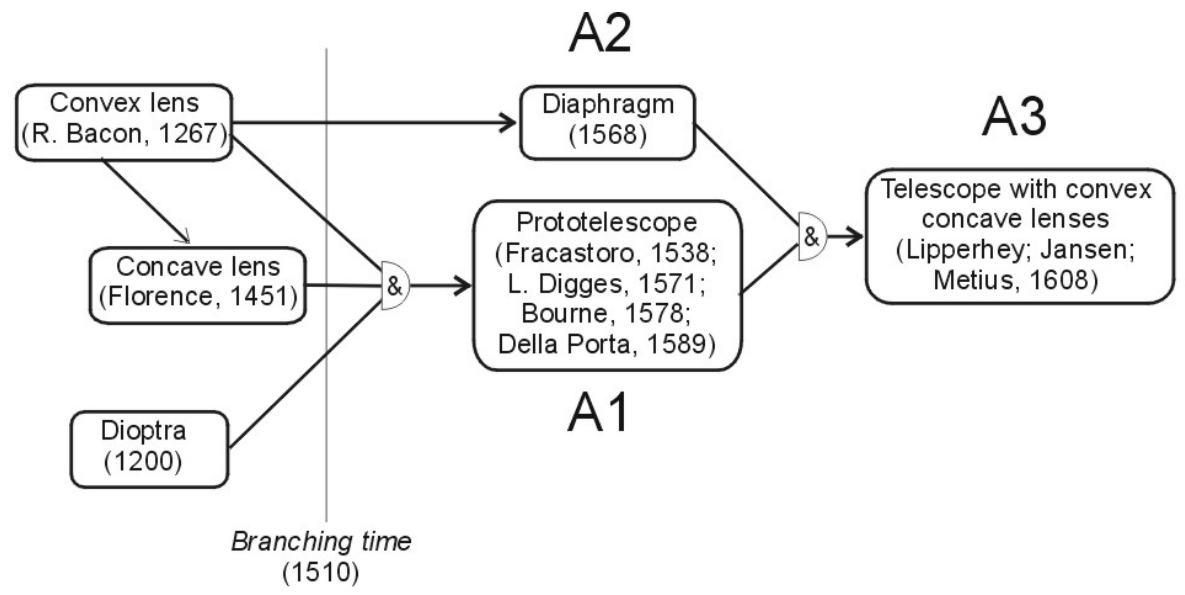

Figure 3: Simplified causal model leading to the invention of the telescope, which may be considered the effect of two previous advances, the prototelescope and the use of a diaphragm. The branching time of 1510 is considered for simulating possible histories of science, maintaining fixed the advances before this time.

Fig. 2b) considering the actual times of the other advances. This results in Fig. 4a, which satisfies PAM. The time at which advance A3 (the invention of the telescope) occurs in $1 \%$ of the possible worlds is 1541 . This indicates that possible world $W$, in which the telescope is taken to have been invented in 1538 is very improbable (a little less than 1\%), given the branching time of 1510 .

Of course, if we had considered a previous branching time, the probability for an early invention of the telescope would increase, as is illustrated in Fig. 4c, which simulates 20,000 worlds branching in 1451 . In this case, the probability for a discovery of the telescope before 1538 rises to around 0.07 (7\%).

\section{Conclusion}

In this paper, we have used two methodological approaches for studying possible worlds in the history of science. The first strategy (section 3) starts from the postulation of a counterfactual world, for whatever reasons the historian of science might encounter, and proposes the use of the principle of the closest possible world (PCP) to guide the construction of the possible world. The second strategy involves computer programming, and offers a method for estimating the probability distribution that a certain advance occurs relative to a previous branching time for the possible worlds being considered. In this latter methodology, the principle that the actual world is the mean (PAM) guides the generation of possible worlds. 

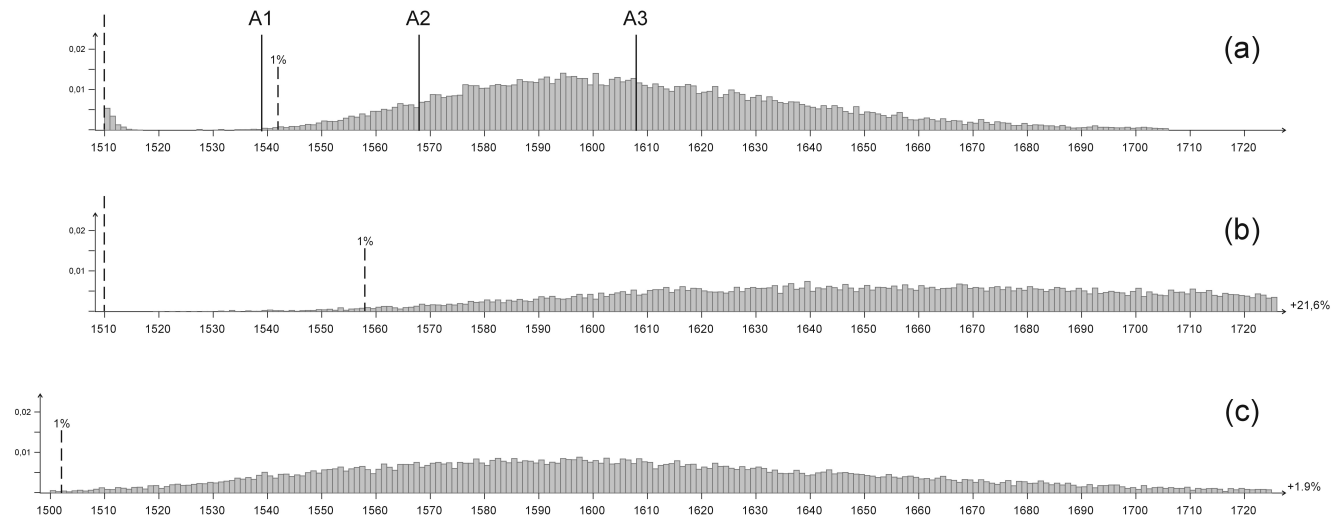

Figure 4: Distribution of the invention of the telescope in one hundred worlds, as possible variations of the model of the actual world (Fig. 3) in which the prototelescope was built in 1538 (A1) and the diaphragm in 1568 (A2). In the actual world, the telescope was invented in 1608 (A3). (a) The time of branching was fixed at 1510, and a simple gamma distribution was computed, as in Fig. 2b, assuming the cause at 1510 and the effect at 1608 . The time in which the invention of the telescope occurs in $1 \%$ of the possible worlds, in this simulation which satisfies PAM (the principle that the actual world is the mean), is 1541. (b) This alternative simulation for branching time at 1510 follows more closely the causal model, in the sense that the dates of the three initial causes of Fig. 3 are maintained (and not brought to 1510, as in Fig.4a), but any world in which A1 or A2 occur before 1510 is discarded. Here the time in which the telescope arises in $1 \%$ of the worlds goes up to 1558 . However, the distribution is skewed to higher values, given that almost half of the simulations were excluded because they gave values for A1 or A2 before 1510 . The mean value is therefore not 1608 , as one would expect from PAM, but around 1662 . There are $21,6 \%$ of the worlds with values after 1725. (c) In this distribution, the time of branching is taken to be 1451, and a procedure similar to Fig.4a was adopted. The gamma distribution centered around 1608 has a much higher standard deviation, and the time in which the telescope appears in $1 \%$ of the worlds comes down to 1502. This illustrates how the chosen branching time affects the distribution of possible worlds.

Principia 20(1): 117-126 (2016). 
Our initial hypothesis that the telescope could have well been invented in 1538 overlooked the importance of the diaphragm in the transition from the prototelescope to the telescope. But the examination of the possible scenarios associated with this hypothesis was not mere handwaving, given the guidance furnished by PCP. We found that the technological antecedence of the telescope would have had only a small influence on the time of discovery that the orbit of Mars is elliptical.

Then we turned to estimate the probability that the invention of the telescope could have occurred in 1538, given a branching time of 1510 . Guided this time by PAM, the result found using computer simulation is that the probability is a little below $0.01(1 \%)$, considering the importance of the use of a diaphragm coupled to the prototelescope. This means that the counterfactual speculations of section 2 are not "realistic" for a branching time of 1510, although they could apply for a counterfactual scenario with a previous branching time, as illustrated in Fig. 4c.

Our calculation of probabilities of possible worlds is based on the hypothesis that the gamma function is a good representation for the probability distribution, with the mean value corresponding to the "empirical" value in our actual world (PAM). However, as discussed in Fig. 2, when there is only one independent occurrence of an effect, we are left with only one datum to fit two unknown constants of the gamma distribution. Thus, an additional hypothesis has been used, fixing the ratio of the standard deviation and the mean of the gamma distribution to the value of the "coefficient of variation" of $c_{v}=\frac{1}{3}$. In future studies, calibrations with situations of independent discoveries, such as that of Fig. 2a, should be able to give a better justification for the value used for $c_{v}$.

The paper is the beginning of studies in computer simulations in the history of science. The resulting paper presents a succession of errors, well in the spirit of Kepler's exploration of the orbit of Mars. But it shows that one of the important features of computational simulations in philosophy of science is to serve as a consistency check for the intuitions and speculations of the philosopher.

\section{Acknowledgements}

We would like to thank Rodrigo de Faria for insightful comments, and also the discussion at the IX Principia International Symposium, especially with Ana Maria Corrêa Moreira da Silva.

\section{References}

Dupré, S. 2003. Galileo's telescope and celestial light. Journal for the History of Astronomy 24: 369-99.

King, H. C. 1955. The history of the telescope. High Wycombe (England): Charles Griffin.

Principia 20(1): 117-126 (2016). 
McCall, S. 1984. Counterfactuals based on real possible worlds. Nous 18: 463-77.

Pessoa Jr., O. 2006. Computation of probabilities in causal models of history of science. Principia 10(2): 109-24.

-2009. Scientific progress as expressed by tree diagrams of possible histories. In: Mortari, C. A. \& Dutra, L. H. A. (eds.) Anais do V Simpósio Internacional Principia (Coleção Rumos da Epistemologia, vol. 9). Florianópolis: NEL-UFSC, p.114-22.

- 2010. Modeling the causal structure of the history of science. In: Magnani, L.; Carnie1li, W. \& Pizzi, C. (eds.). Model-based reasoning in science and technology. Berlin: Springer, Berlin, p.643-54.

- 2014. Como construir um mundo causalmente possível? Ideação 29: 13-30.

Van Helden, A. 1977. The invention of the telescope. Transactions of the American Philosophical Society 67: 1-67.

Willach, R. 2010. The long road to the invention of the telescope. In: A. Van Helden; S. Dupré; R. van Gent; H. Zuidervaart (eds.) The origins of the telescope, p.93-114. Amsterdam: KNAW Press.

Zuidervaart, H. J. 2010. The 'true inventor' of the telescope. A survey of 400 years of debate. In: A. Van Helden; S. Dupré; R. van Gent; H. Zuidervaart (eds.) The origins of the telescope, p.9-44. Amsterdam: KNAW Press.

Osvaldo Pessoa JR. Department of Philosophy University of São Paulo opessoa@usp.br

RAFAELA GESING

Undergraduate student Institute of Mathematics and Statictics

University of São Paulo rafaelagesing@gmail.com

MARIANA Jó DE SOUZA

Undergraduate student Institute of Physics University of São Paulo mariana.jsouza@gmail.com

Daniel Carlos de Melo Marcílio

Undergraduate student

Polytechnic School

University of São Paulo daniel.marcilio@usp.br

Principia 20(1): 117-126 (2016). 